\title{
Identification of Key Genes Related with Alzheimer's Disease Treatment Through Bioinformatics Analysis
}

\author{
Pan Liu \\ Laboratory of Biochemical Engineering, College of Pharmaceutical Engineering and \\ Life Sciences, Changzhou University, Changzhou 213003, China \\ E-mail: qinpanliu@163.com
}

Hongjia Qian

Laboratory of Biochemical Engineering, College of Pharmaceutical Engineering and

Life Sciences, Changzhou University, Changzhou 213003, China

E-mail: 1397862340@qq.com

Liqun Wang (Corresponding author)

Laboratory of Biochemical Engineering, College of Pharmaceutical Engineering and

Life Sciences, Changzhou University, Changzhou 213003, China

Tel: +86-0519-86334597; E-mail: wlq@cczu.edu.cn

Received: January 13, 2018 Accepted: February 1, 2018

doi:10.5296/jbls.v9i1.12474 URL: https://doi.org/10.5296/jbls.v9i1.12474

\begin{abstract}
T0901317, a live $X$ receptor agonist, can reduce amyloid $\beta$ generation in vitro and in a mouse Alzheimer's disease (AD) model. To investigate the global molecular effects of T0901317 in mouse hippocampus, we downloaded public GSE31624 generated from the hippocampus of wild-type mice, Tg2576 mice and T0901317-treated Tg2576 mice. Differentially-expressed genes (DEGs) were identified on LIMMA of $\mathrm{R}$ software. Gene ontology and Kyoto Encyclopedia of Genes and Genomes enrichment were analyzed through DAVID. Proteinprotein interaction and hub genes were obtained based on STRING and Cytoscape. Nine downregulated and 68 upregulated DEGs in T0901317-treated Tg2576 were identified in comparison with untreated Tg2576 mice. Annotation analyses showed these DEGs correlated
\end{abstract}


with transport (BP), membrane (CC) and binding (MF) terms and the dopaminergic synapse pathway. Protein-protein interaction network was built to find out some hub genes by maximal clique centrality. Discs large homolog 4 (Dlg4), the most outstanding gene, was associated with cognition improvement in aged AD mice. T0901317 may impact the development by regulating the Dlg4 expression. In conclusion, we investigated effects of T0901317 therapy on gene expression profiles in the hippocampus of Tg2576 mice and found Dlg4 may serve as putative therapeutics target for $\mathrm{AD}$ treatment.

Keywords: T0901317, Alzheimer's disease, differentially-expressed genes, enrichment analysis

\section{Introduction}

Alzheimer's disease (AD), the major form of dementia in the elderly, is featured by amyloid plaques outside cerebral cells and neurofibrillary tangles inside cerebral cells (Henderson, 2014). Dementia has affected up to 24 million people worldwide and attacks 4.6 million new cases every year. About $70 \%$ of these cases developed to AD (Reitz \& Mayeux, 2014). The growing number of patients, long-term disability, and the rising AD care costs have become public health concerns (Sibener et al., 2014). Therefore, early diagnosis and treatment of AD patients are urgent.

Experimental evidence reveals changes in lipid synthesis and metabolism are dysfunctional in the AD brain, which could raise the risk of AD development (Garner, 2010). Apolipoprotein $\mathrm{E}(\mathrm{ApoE})$ is a versatile protein playing critical roles in neurobiology, lipid metabolism, and neurodegeneration (Huang \& Mahley, 2014). Low plasma ApoE level is associated with smaller hippocampal size (Teng et al., 2015). ATP-binding cassette subfamily A member 1 (ABCA1) is needed to normalize ApoE levels in the central nervous system and lipidize astrocyte-secreted ApoE (Wahrle et al., 2004). ABCA1 overexpression reduces amyloid beta-peptide $(A \beta)$ deposition in a mouse AD model (Wahrle et al., 2008). Liver $X$ receptor (LXR) not only inhibits amyloid precursor protein (APP) processing by modulating membrane cholesterol levels via ABCA1, but also accelerates the clearance of $A \beta$ by inducing ApoE lipoprotein secretion (Riddell et al., 2007). Further, the LXR agonist T0901317 decreases A $\beta$ generation in vitro and in a mouse AD model (Koldamova et al., 2005). However, analyses of the transcriptomic response of mouse hippocampus to T0901317 remain unclear.

In this study, we downloaded public microarray data (GSE31624) to find out differentially expressed genes (DEGs) in the brain of APPswe transgenic mice (Tg2576) and T0901317-treated Tg2576 mice. The functions of DEGs were assessed by annotation, pathway enrichment and protein-protein interaction (PPI) establishment. This may provide insight into the effect of T0901317 on the hippocampus of Tg2576 mice and identify gene expression signatures for prediction of therapeutic effects of T0901317. 


\section{Materials and Methods}

\subsection{Public microarray data}

The gene expression profile (GSE31624) was downloaded from the gene expression omnibus (GEO) (www.ncbi.nlm.nih.gov/geo) based on platform GPL1261 (Affymetrix Mouse Genome U133 Plus 2.0 Array). Follettie et al. deposited the dataset and explored how the hippocampus of wild-type mice and $\mathrm{Tg} 2576$ mice responded to T0901317. Totally 33 samples were analyzed (wild type, $\operatorname{Tg} 2576$ mice, and T090131-treated $\operatorname{Tg} 2576$ mice each with 11 replicates).

The gene expression profile was calibrated, quantile-normalized and probe-summarized using the Robust Multi-Array Average algorithm in Affy of Bioconductor (www.bioconductor.org/).

\subsection{Identification of DEGs}

DEGs were identified using Linear Models for Microarray Data package (LIMMA, www.biocondutor.org/packages/release/bioc/html/limma.html) (Ritchie et al., 2015) in Bioconductor compared in the T0901317-treated Tg2576 mice. P < 0.05 (Z. Lin \& Lin, 2017; Zhang et al., 2017) and $\mid \log 2$ (fold change) $\mid \geq 1.5$ were considered as the threshold.

\subsection{Functional enrichment analysis of DEGs}

Gene Ontology (GO) function and Kyoto Encyclopedia of Genes and Genomes (KEGG) pathway enrichment of DEGs were analyzed in Database for Annotation, Visualization and Integrated Discovery (DAVID), which offers a complete set of function annotation tools to determine the biological information behind a large list of genes (Huang da, Sherman, \& Lempicki, 2009). The cut-off criterion was $\mathrm{P}<0.05$.

\subsection{PPI network establishment}

STRING (www.string-db.org/) offers an important evaluation and integration of PPI, including direct (physical) and indirect (functional) associations (Szklarczyk et al., 2015). PPIs of DEGs with score (median confidence) $>0.4$ were chosen, and PPI network was visualized by Cytoscape (www.cytoscape.org/). Hub Object Analyzer (Hubba), a web-based tool, explores important nodes in an interactome network established from specific small- or large-scale methods based on graph theory (C. Y. Lin et al., 2008). Hubba in cytoscape was applied to find important nodes.

\section{Results}

\subsection{Identification of DEGs}

Based on our selection criteria, 55 DEGs (35 downregulated and 20 upregulated DEGs) were obtained in $\mathrm{Tg} 2576$ mice. In addition, 77 DEGs altered in T0901317-treated Tg2576 mice compared with untreated mice ( 9 downregulated and 68 upregulated). The top 10 downregulated and upregulated DEGs were summarized in Table 1. The hierarchical clustering of expression data (T0901317 vs. untreated) was shown in Figure 1. Notably, 22 genes were similarly abnormally expressed between groups (Table 2). 


\section{IIMacrothink}

Journal of Biology and Life Science

ISSN 2157-6076 2018, Vol. 9, No. 1

Table 1. The top 10 significant up-regulated and down-regulated differential expressed genes (Tg2576 mice vs. Wild type and TO901317 treated vs. untreated Tg2576)

\begin{tabular}{|c|c|c|c|c|c|c|c|}
\hline \multicolumn{4}{|c|}{ Tg2576 mice vs. Wild type } & \multicolumn{4}{|c|}{ T0901317 treated vs. untreated $\mathrm{Tg} 2576$} \\
\hline Probe set & Gene symbol & $\begin{array}{l}\text { Log2(Fold } \\
\text { change) }\end{array}$ & P value & Probe set & Gene symbol & $\begin{array}{l}\text { Log } 2(\text { Fold } \\
\text { change })\end{array}$ & $P$ value \\
\hline \multicolumn{4}{|l|}{ Down-regulated } & \multicolumn{4}{|l|}{ Up-regulated } \\
\hline 1450407_a_at & Anp32a & -1.6221 & 0.001492 & 1431708_a_at & Tia1 & -1.52435 & 0.016148 \\
\hline 1417727_at & Srsf9 & -1.0523 & 0.023495 & 1439483_at & AI506816 & -1.48716 & $3.98 \mathrm{E}-05$ \\
\hline 1419093_at & Tdo2 & -1.03976 & $1.27 \mathrm{E}-05$ & 1424454_at & Tmem87a & -0.97678 & 0.014682 \\
\hline 1437125_at & Camk2a & -0.97836 & 0.001428 & 1418148_at & Abhd1 & -0.89505 & $2.53 \mathrm{E}-05$ \\
\hline 1419672_at & Spock1 & -0.96861 & 0.018757 & 1439627_at & Zic1 & -0.83383 & 0.019484 \\
\hline 1447831_s_at & Mtmr7 & -0.94316 & 0.041899 & 1450779_at & Fabp7 & -0.75755 & 0.001782 \\
\hline 1435367_at & Mapk4 & -0.92343 & 0.016527 & 1431328_at & Ppp1cb & -0.69389 & 0.000144 \\
\hline 1419580_at & Dlg4 & -0.90936 & 0.025875 & 1460310_a_at & Gh & -0.66959 & 0.030909 \\
\hline 1437183_at & Lrrc $4 b$ & -0.87873 & 0.041879 & 1435602_at & Sephs2 & -0.62408 & 0.021469 \\
\hline 1417184_s_at & Hbb-b1/b2/bs/bt & -0.85775 & 0.022617 & 1426288_at & Lrp4 & 0.585653 & 0.014084 \\
\hline \multicolumn{8}{|l|}{ Up-regulated } \\
\hline 1449254_at & Spp1 & 0.922053 & 0.010075 & 1450407_a_at & Anp32a & 1.389023 & 0.006343 \\
\hline 1418395_at & Slc47a1 & 0.880921 & 0.006401 & 1432646_a_at & 2900097C17Rik & 1.275417 & 0.023875 \\
\hline 1447808_s_at & Slc15a2 & 0.764701 & 0.028212 & 1425963_at & Cabp7 & 1.079491 & 0.019929 \\
\hline 1453287_at & Ankrd33b & 0.719801 & 0.046855 & 1435367_at & Mapk4 & 1.073758 & 0.006901 \\
\hline 1417933_at & Igfbp6 & 0.717806 & $3.74 \mathrm{E}-05$ & 1419672_at & Spock1 & 1.004938 & 0.014851 \\
\hline 1436996_x_at & Lyz1 & 0.691264 & 0.000791 & 1460717_at & Tspyl1 & 0.963865 & 0.041016 \\
\hline 1435602_at & Sephs2 & 0.688338 & 0.014488 & 1437125_at & Camk2a & 0.963662 & 0.0016 \\
\hline 1416625_at & Serping1 & 0.687692 & 0.007146 & 1415801_at & Gja1 & 0.954418 & 0.043414 \\
\hline 1434447_at & Met & 0.669609 & $4.28 \mathrm{E}-05$ & 1419580_at & $\operatorname{Dlg} 4$ & 0.947123 & 0.018229 \\
\hline 1416953_at & Ctgf & 0.665987 & 0.022056 & 1437183_at & Lrrc4b & 0.941328 & 0.029787 \\
\hline
\end{tabular}



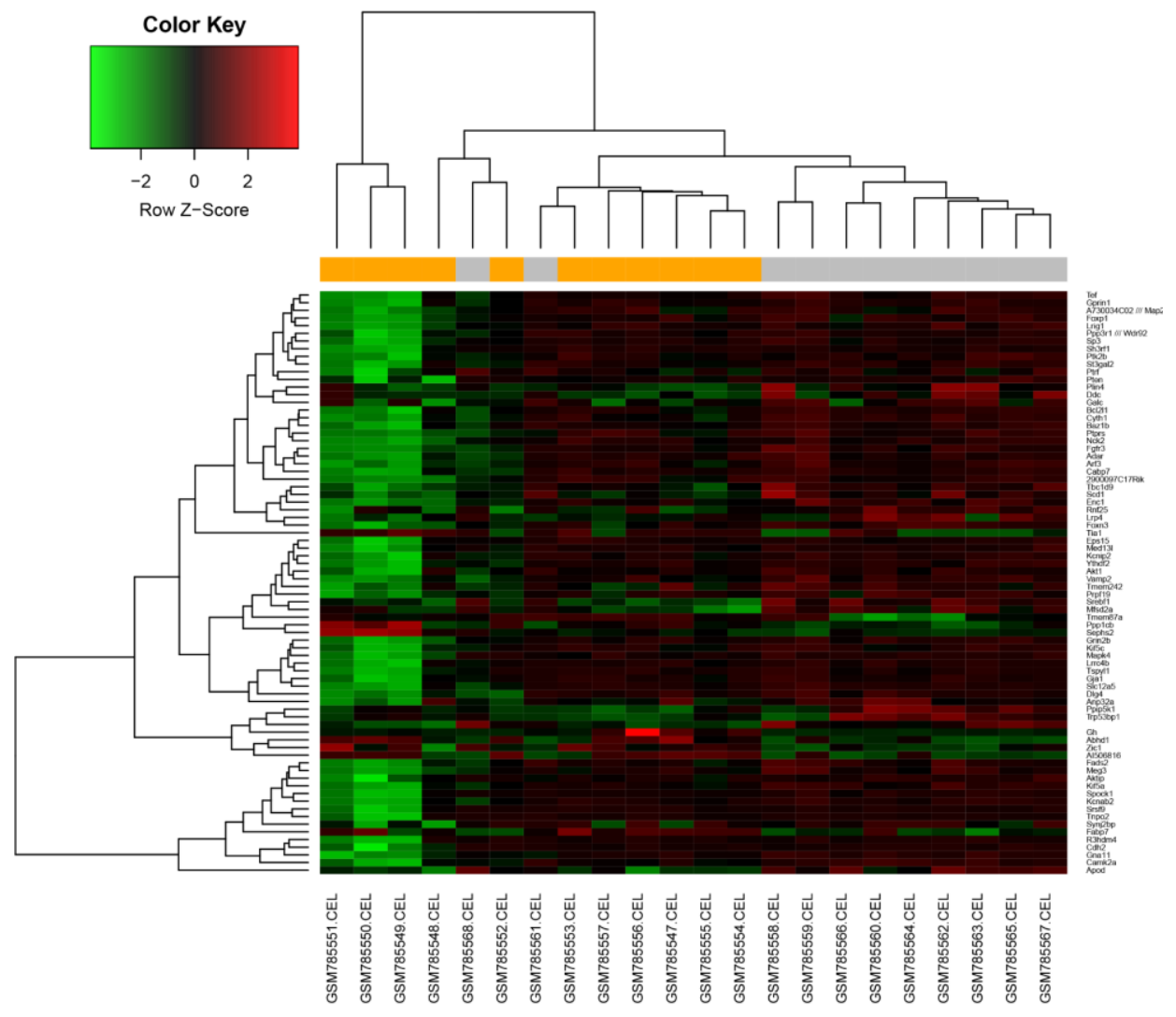

Figure 1. Heatmap for differentially expressed genes (DEGs). Grey and yellow color represents cell culture of pericyte without/with T0901317 respectively. The color bar denotes z-score adjusted expression values, green used for down-regulation and red for up-regulation. 


\section{Macrothink}

Journal of Biology and Life Science

ISSN 2157-6076 2018, Vol. 9, No. 1

Table 2. Similar abnormal expression of genes in $\mathrm{Tg} 2576$ mice and mice treated with T0901317.

\begin{tabular}{llcc}
\hline ID & $\begin{array}{l}\text { Gene } \\
\text { symbol }\end{array}$ & $\begin{array}{l}\text { Log2(Fold change) } \\
\text { vs. wild type) }\end{array}$ & $\begin{array}{c}\text { Log2(Fold change) (T0901317 } \\
\text { vs. untreated) }\end{array}$ \\
\hline 1426288_at & Lrp4 & -0.79999 & 0.585653 \\
1423477_at & Zic1 & 0.626141 & -0.83383 \\
1437283_at & Tnpo2 & -0.83092 & 0.848281 \\
1437125_at & Camk2a & -0.97836 & 0.963662 \\
1435602_at & Sephs2 & 0.688338 & -0.62408 \\
1449144_at & Gna11 & -0.6561 & 0.642909 \\
1450407_a_at & Anp32a & -1.6221 & 1.389023 \\
1448997_at & Cyth1 & -0.60585 & 0.6951 \\
1425870_a_at & Kcnip2 & -0.68441 & 0.63504 \\
1417727_at & Srsf9 & -1.0523 & 0.939293 \\
1419672_at & Spock1 & -0.96861 & 1.004938 \\
1435367_at & Mapk4 & -0.92343 & 1.073758 \\
1433932_x_at & R3hdm4 & -0.78515 & 0.740296 \\
1418815_at & Cdh2 & -0.80634 & 0.859129 \\
1431804_a_at & Sp3 & -0.80203 & 0.792424 \\
1423364_a_at & Aktip & -0.67833 & 0.635873 \\
1438255_at & Foxn3 & -0.77672 & 0.633855 \\
1437183_at & Lrrc4b & -0.87873 & 0.941328 \\
1442370_at & Grin2b & -0.79605 & 0.867629 \\
1419580_at & Dlg4 & -0.90936 & 0.947123 \\
1434602_at & Med131 & -0.63042 & 0.736594 \\
1424045_at & Tmem242 & -0.64295 & 0.605184 \\
\hline
\end{tabular}

\subsection{Enrichment analyses of DEGs}

Our team tried to explore the changed biofunctions of the DEGs by GO and KEGG analysis in DAVID. The enriched GO terms divided into cellular component (CC), bioprocess (BP) and molecular function (MF) was illustrated as Figure 2. The DEGs (Tg2576 vs. wild-type) were associated with transport (BP), membrane (CC) and binding (MF) and the pathway of malaria.

For T0901317-treated Tg2576 mice, DEGs were mainly involved in transport, such as transport (13 genes), apoptotic process (9 genes) and lipid metabolic process ( 6 genes) in the $\mathrm{BP}$ ontology. In the CC ontology, the most significant GO categories were membrane- related, such as membrane (38 genes), plasma membrane ( 28 genes), and nucleoplasm (14 genes). In the MF ontology, the binding terms accounted for the majority of enriched GO categories, including protein binding (35 genes), protein complex binding (8 genes) and protein kinase binding (8 genes). 


\section{Macrothink Institute ${ }^{\mathrm{TM}}$}

KEGG pathway enrichment analysis indicated the DEGs were mainly enriched in pathways of insulin resistance (e.g. SREBF1, PTEN and PPP1CB), long-term potentiation (e.g. GRIN2B, PPP3R1 and CAMK2A) and dopaminergic synapse (e.g. AKT1, DDC and GRIN2B) (Table 3).

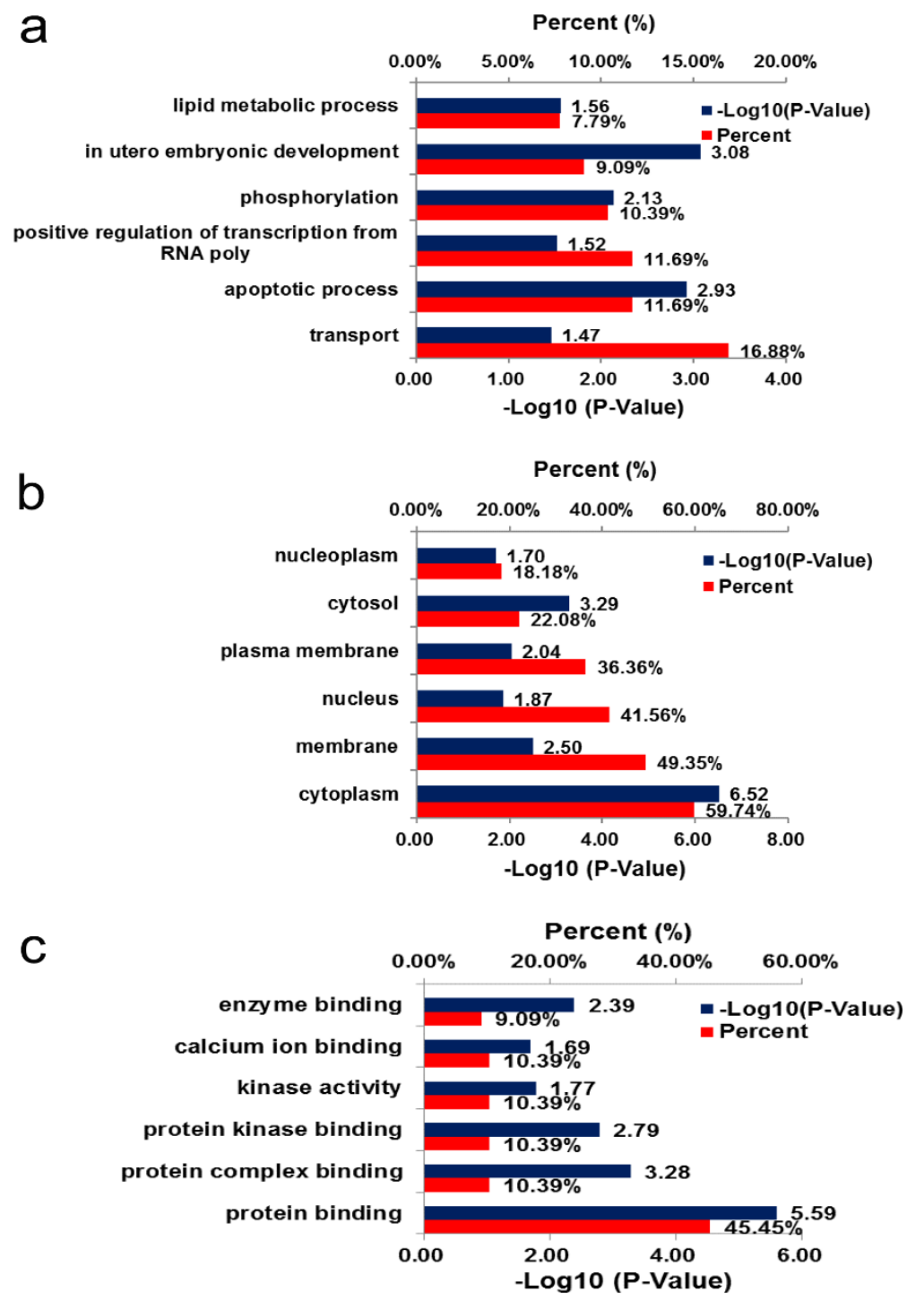

Figure 2. GO enrichment of DEGs. a, biological process (BP); b, cellular component (CC); c, molecular function (MF).

Table 3. The enriched KEGG pathway of differential expression genes.

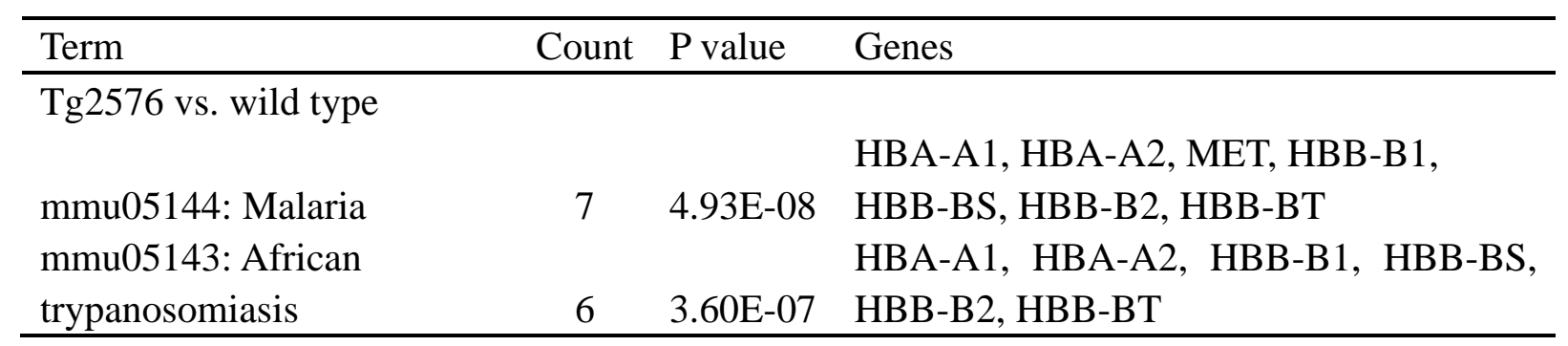


mmu04510: Focal adhesion

mmu04512: ECM-receptor

interaction

mmu05030: Cocaine

addiction

T0901317 vs. untreated

mmu04728: Dopaminergic

synapse

mmu04144: Endocytosis

mmu05031: Amphetamine

addiction

mmu04720: Long-term

potentiation

mmu04931: Insulin

resistance
$5 \quad 0.012406 \quad$ SPP1

40.006636 COL3A1, COL1A2, COL6A1, SPP1

$3 \quad 0.019456$ GRM3, GRIN2B, DLG4

AKT1, DDC, GRIN2B, KIF5A, KIF5C,

7 2.21E-05 CAMK2A, PPP1CB

EPS15, FGFR3, CYTH1, KIF5A, ARF3,

$6 \quad 0.006899$ KIF5C

DDC, GRIN2B， PPP3R1， CAMK2A,

5 1.98E-04 PPP1CB

40.002956 GRIN2B, PPP3R1, CAMK2A, PPP1CB

$4 \quad 0.012221 \quad$ SREBF1, AKT1, PTEN, PPP1CB

\subsection{PPI network analysis}

Totally 77 DEGs (T0901317-treated vs. Tg2576) were sent to STRING for establishment of PPI networks (combined score > 0.4) (Figure 3). The hub genes evaluated by betweenness maximal clique centrality (MCC), which showed the most important gene was discs large MAGUK scaffold protein 4 (Dlg4).

a

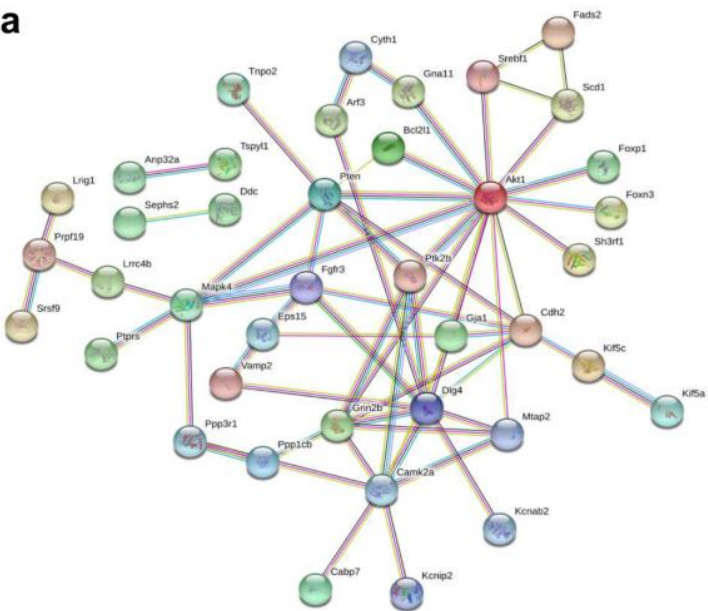

b

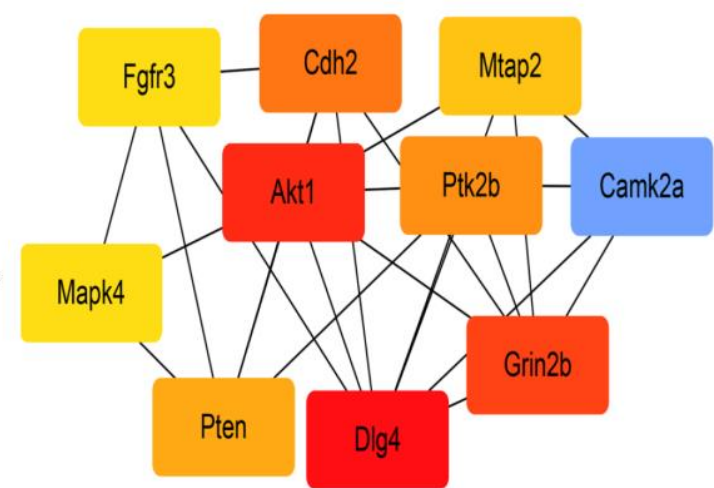

Figure 3. Protein-protein network of DEGs altered in $\mathrm{Tg} 2576$ mice treated with T0901317. a, string database; $b$, the hub genes identified from protein-protein network. 


\section{Discussion}

Our group analyzed the bioinformatics of gene expression profiles from $\mathrm{Tg} 2576$ mice and T0901317-treated Tg2576 mice to explore the mechanisms of T0901317 therapy on AD. Then 68 up-regulated DEGs and 9 down-regulated DEGs were obtained. Annotation results indicated these DEGs correlated with transport (BP), membrane (CC) and binding (MF) terms and the pathway of dopaminergic synapse. Totally 10 crucial genes were identified in the PPI model and the most outstanding gene was Dlg4.

$\mathrm{AD}$, the dominant cause and common form of dementia, is featured by a gradual decent in cognition, which occurs with memory degradation (Gurav, 2014). $\mathrm{Tg} 2576$ mice are the common animal models of AD (Sooy et al., 2015). A Tg2576 mouse model of AD showed T0901317 selectively reduced hippocampal A $\beta 42$ level and enhanced memory (Riddell et al., 2007). We explored how $\mathrm{Tg} 2576$ hippocampus responded to T0901317 from the transcriptomic perspective.

We obtained 55 DEGs (Tg2576 vs. wild) and 77 DEGs (T0901317 vs. untreated) according to our selection criteria. In Table 2, 22 genes were similarly abnormally expressed between Tg2576/wild-type groups and T0901317/untreated groups. Low-density lipoprotein receptorrelated protein 4 (Lrp4) is required to normalize hippocampal plasticity and maintain longterm potentiation (Pohlkamp et al., 2015). The improved cognitive functions could be due to the regulation of the $\gamma$-aminobutyric acid receptor system, upregulation of microtubuleassociated protein 2, and inhibition of N-cadherin cleavage (Mishra \& Heese, 2011). Much evidence shows glutamate receptor, ionotropic, NMDA2B epsilon 2 (Grin2b) could be involved in specific function pertaining to the expressing cells, and its mRNA levels were significantly downregulated in these susceptible regions of the AD brain (Andreoli et al., 2014). These DEGs were down-regulated in Tg2576 mice, but up-regulated in the T090137treated $\mathrm{Tg} 2576$. So far, no study reported the link between the remaining genes and AD.

Pathway analyses indicated the DEGs (T0901317 vs. untreated) were typically enriched in pathways of dopaminergic synapse, long-term potentiation and insulin resistance. Some results are compatible with previous studies. Long-term potentiation is critical in AD development (Koch et al., 2012; Wu, Guo, Gearing, \& Chen, 2014). Brain insulin resistance is closely correlated to cognitive degradation and promotes biological abnormality in the disorder (Talbot, 2014). Abnormal insulin signaling contributes to synaptic and memory deteriorations (De Felice, Lourenco, \& Ferreira, 2014). A single dose of oral L-dopa, one key neurotransmitter in regulating synaptic plasticity mechanisms, induced a clear form of reversal of the direction of plasticity in probable AD (Koch et al., 2011). However, there is rare research on dopaminergic synapse.

Ten hub genes (Dlg4, Akt1, Grin2b, Cdh2, Ptk2b, Pten, Mapk4, Fgfr3, Mtap2, Camk2a) were obtained based on MCC in the PPI network. Reactive oxygen species-regulated oxidative correction of Akt1 accounts for the loss of activity-dependent protein translation that is critical for synaptic plasticity and maintenance (Ahmad et al., 2017). Ptk2b gene polymorphisms increase the risk of late-onset AD (Beck, Nicolas, Kopp, \& Golemis, 2014). Pten inhibition saved synaptic function and cognition in $\mathrm{AD}$ animals, while Pten 
overexpression was synaptically depressed, which mimicked and prevented A $\beta$-induced depression (Knafo et al., 2016). Postsynaptic density protein 95 (PSD95), transcribed from the Dlg4 (the most outstanding gene in the PPI network), mediated the expressions of important neuron-related protein- coding genes involved in neuronal plasticity and memory (Bustos et al., 2017). The aberrance of PSD95 expression in AD limited plasticity and stabilized the neuronal circuitry (Bustos et al., 2017). Dlg4/PSD95 will become a new target for the treatment.

This study has several limitations First, gene expression profiles were not validated experimentally due to limited resource. Second, the number of mouse hippocampus was small, which may make our study underpowered. Third, the public microarray data were from mouse, and might not apply to humans.

\section{Conclusion}

Microarrays were used to find out gene expression modulated in the hippocampus of $\operatorname{Tg} 2576$ mice and the transcriptional response to treatment with an LXR agonist T0901317. It is beneficial to exploring the mechanism of T0901317 on the mouse hippocampus. However, the findings should be confirmed by larger studies.

\section{References}

Ahmad, F., Singh, K., Das, D., Gowaikar, R., Shaw, E., Ramachandran, A., . . Ravindranath, V. (2017). Reactive Oxygen Species-Mediated Loss of Synaptic Akt1 Signaling Leads to Deficient Activity-Dependent Protein Translation Early in Alzheimer's Disease. Antioxid Redox Signal, 27(16), 1269-1280. doi:10.1089/ars.2016.6860

Andreoli, V., De Marco, E. V., Trecroci, F., Cittadella, R., Di Palma, G., \& Gambardella, A. (2014). Potential involvement of GRIN2B encoding the NMDA receptor subunit NR2B in the spectrum of Alzheimer's disease. J Neural Transm (Vienna), 121(5), 533-542.

doi:10.1007/s00702-013-1125-7

Beck, T. N., Nicolas, E., Kopp, M. C., \& Golemis, E. A. (2014). Adaptors for disorders of the brain? The cancer signaling proteins NEDD9, CASS4, and PTK2B in Alzheimer's disease. Oncoscience, 1(7), 486-503. doi:10.18632/oncoscience.64

Bustos, F. J., Ampuero, E., Jury, N., Aguilar, R., Falahi, F., Toledo, J., . . . van Zundert, B. (2017). Epigenetic editing of the Dlg4/PSD95 gene improves cognition in aged and Alzheimer's disease mice. Brain, 140(12), 3252-3268. doi:10.1093/brain/awx272

De Felice, F. G., Lourenco, M. V., \& Ferreira, S. T. (2014). How does brain insulin resistance develop in Alzheimer's disease? Alzheimers Dement, 10(1 Suppl), S26-32.

doi:10.1016/j.jalz.2013.12.004

Garner, B. (2010). Lipids and Alzheimer's disease. Biochim Biophys Acta, 1801(8), 747-749. doi:10.1016/j.bbalip.2010.06.003

Gurav, A. N. (2014). Alzheimer's disease and periodontitis--an elusive link. Rev Assoc Med Bras (1992), 60(2), 173-180. Retrieved from http://www.ncbi.nlm.nih.gov/pubmed/24919005 
Henderson, V. W. (2014). Alzheimer's disease: review of hormone therapy trials and implications for treatment and prevention after menopause. J Steroid Biochem Mol Biol, 142, 99-106. doi:10.1016/j.jsbmb.2013.05.010

Huang da, W., Sherman, B. T., \& Lempicki, R. A. (2009). Systematic and integrative analysis of large gene lists using DAVID bioinformatics resources. Nat Protoc, 4(1), 44-57. doi:10.1038/nprot.2008.211

Huang, Y., \& Mahley, R. W. (2014). Apolipoprotein E: structure and function in lipid metabolism, neurobiology, and Alzheimer's diseases. Neurobiol Dis, 72 Pt A, 3-12.

doi:10.1016/j.nbd.2014.08.025

Knafo, S., Sanchez-Puelles, C., Palomer, E., Delgado, I., Draffin, J. E., Mingo, J., . . Esteban, J. A. (2016). PTEN recruitment controls synaptic and cognitive function in Alzheimer's models. Nat Neurosci, 19(3), 443-453. doi:10.1038/nn.4225

Koch, G., Di Lorenzo, F., Bonni, S., Ponzo, V., Caltagirone, C., \& Martorana, A. (2012). Impaired LTP- but not LTD-like cortical plasticity in Alzheimer's disease patients. J Alzheimers Dis, 31(3), 593-599. doi:10.3233/JAD-2012-120532

Koch, G., Esposito, Z., Codeca, C., Mori, F., Kusayanagi, H., Monteleone, F., . . Martorana, A. (2011). Altered dopamine modulation of LTD-like plasticity in Alzheimer's disease patients. Clin Neurophysiol, 122(4), 703-707. doi:10.1016/j.clinph.2010.10.033

Koldamova, R. P., Lefterov, I. M., Staufenbiel, M., Wolfe, D., Huang, S., Glorioso, J. C., . . . Lazo, J. S. (2005). The liver X receptor ligand T0901317 decreases amyloid beta production in vitro and in a mouse model of Alzheimer's disease. J Biol Chem, 280(6), 4079-4088.

doi:10.1074/jbc.M411420200

Lin, C. Y., Chin, C. H., Wu, H. H., Chen, S. H., Ho, C. W., \& Ko, M. T. (2008). Hubba: hub objects analyzer--a framework of interactome hubs identification for network biology. Nucleic Acids Res, 36(Web Server issue), W438-443. doi:10.1093/nar/gkn257

Lin, Z., \& Lin, Y. (2017). Identification of potential crucial genes associated with steroid-induced necrosis of femoral head based on gene expression profile. Gene, 627, 322-326. doi:10.1016/j.gene.2017.05.026

Mishra, M., \& Heese, K. (2011). P60TRP interferes with the GPCR/secretase pathway to mediate neuronal survival and synaptogenesis. J Cell Mol Med, 15(11), 2462-2477.

doi:10.1111/j.1582-4934.2010.01248.x

Pohlkamp, T., Durakoglugil, M., Lane-Donovan, C., Xian, X., Johnson, E. B., Hammer, R. E., \& Herz, J. (2015). Lrp4 domains differentially regulate limb/brain development and synaptic plasticity. PLoS One, 10(2), e0116701. doi:10.1371/journal.pone.0116701

Reitz, C., \& Mayeux, R. (2014). Alzheimer disease: epidemiology, diagnostic criteria, risk factors and biomarkers. Biochem Pharmacol, 88(4), 640-651. doi:10.1016/j.bcp.2013.12.024

Riddell, D. R., Zhou, H., Comery, T. A., Kouranova, E., Lo, C. F., Warwick, H. K., . . . 
Jacobsen, J. S. (2007). The LXR agonist TO901317 selectively lowers hippocampal Abeta42 and improves memory in the Tg2576 mouse model of Alzheimer's disease. Mol Cell Neurosci, 34(4), 621-628. doi:10.1016/j.mcn.2007.01.011

Ritchie, M. E., Phipson, B., Wu, D., Hu, Y., Law, C. W., Shi, W., \& Smyth, G. K. (2015). limma powers differential expression analyses for RNA-sequencing and microarray studies. Nucleic Acids Res, 43(7), e47. doi:10.1093/nar/gkv007

Sibener, L., Zaganjor, I., Snyder, H. M., Bain, L. J., Egge, R., \& Carrillo, M. C. (2014). Alzheimer's Disease prevalence, costs, and prevention for military personnel and veterans. Alzheimers Dement, 10(3 Suppl), S105-110. doi:10.1016/j.jalz.2014.04.011

Sooy, K., Noble, J., McBride, A., Binnie, M., Yau, J. L., Seckl, J. R., . . Webster, S. P. (2015). Cognitive and Disease-Modifying Effects of 11beta-Hydroxysteroid Dehydrogenase Type 1 Inhibition in Male Tg2576 Mice, a Model of Alzheimer's Disease. Endocrinology, 156(12), 4592-4603. doi:10.1210/en.2015-1395

Szklarczyk, D., Franceschini, A., Wyder, S., Forslund, K., Heller, D., Huerta-Cepas, J., . . . von Mering, C. (2015). STRING v10: protein-protein interaction networks, integrated over the tree of life. Nucleic Acids Res, 43(Database issue), D447-452. doi:10.1093/nar/gku1003

Talbot, K. (2014). Brain insulin resistance in Alzheimer's disease and its potential treatment with GLP-1 analogs. Neurodegener Dis Manag, 4(1), 31-40. doi:10.2217/nmt.13.73

Teng, E., Chow, N., Hwang, K. S., Thompson, P. M., Gylys, K. H., Cole, G. M., . . . Alzheimer's Disease Neuroimaging, I. (2015). Low plasma ApoE levels are associated with smaller hippocampal size in the Alzheimer's disease neuroimaging initiative cohort. Dement Geriatr Cogn Disord, 39(3-4), 154-166. doi:10.1159/000368982

Wahrle, S. E., Jiang, H., Parsadanian, M., Kim, J., Li, A., Knoten, A., . . Holtzman, D. M. (2008). Overexpression of ABCA1 reduces amyloid deposition in the PDAPP mouse model of Alzheimer disease. J Clin Invest, 118(2), 671-682. doi:10.1172/JCI33622

Wahrle, S. E., Jiang, H., Parsadanian, M., Legleiter, J., Han, X., Fryer, J. D., . . Holtzman, D. M. (2004). ABCA1 is required for normal central nervous system ApoE levels and for lipidation of astrocyte-secreted apoE. J Biol Chem, 279(39), 40987-40993.

doi:10.1074/jbc.M407963200

Wu, Z., Guo, Z., Gearing, M., \& Chen, G. (2014). Tonic inhibition in dentate gyrus impairs long-term potentiation and memory in an Alzheimer's [corrected] disease model. Nat Commun, 5, 4159. doi:10.1038/ncomms5159

Zhang, L., Huang, Y., Zhuo, W., Zhu, Y., Zhu, B., \& Chen, Z. (2017). Identification and characterization of biomarkers and their functions for Lapatinib-resistant breast cancer. Med Oncol, 34(5), 89. doi:10.1007/s12032-017-0953-y 


\section{Copyright Disclaimer}

Copyright for this article is retained by the author(s), with first publication rights granted to the journal.

This is an open-access article distributed under the terms and conditions of the Creative Commons Attribution license (http://creativecommons.org/licenses/by/3.0/). 